\title{
What's new in abstracts of science articles?
}

\author{
James Hartley, PhD
}

See end of article for author's affiliation.

DOI: http://dx.doi.org/10.3163/1536-5050.104.3.011

In 2004 [1], the author reported the then current findings from research on structured abstracts and followed this up with a similar paper in 2014 [2]. This present commentary updates both of these earlier ones and demonstrates that research on the design of abstracts continues apace. Here, I comment on developments in graphical, video, readable, and tweetable abstracts.

\section{GRAPHICAL ABSTRACTS}

More journals are beginning to include graphical abstracts in addition to their traditional ones. Such abstracts are supposed to provide "a single, concise pictorial and visual summary of the main findings of an article." To my mind, however, these abstracts are often difficult to understand and one has to appreciate the text beforehand to understand the graphic! Current life sciences journals advocating and using graphical abstracts include the International Journal of Pharmaceutics and the Journal of Ethnopharmacology. Elsevier's web page "Graphical Abstracts" $<$ https:www.elsevier.com/authors/journal-authors/ graphical-abstract $>$ provides sixteen examples of good graphic abstracts in published articles.

\section{VIDEO ABSTRACTS}

Short (five-minute) video abstracts allow authors to present their research in person to the reader. They are usually created after a paper has been accepted and are linked to the paper on publication. Normally presented by the main authors, sometimes using animation and infographics, these abstracts provide a welcome introduction to the aims and findings of the paper that follows. Examples can be found in electronic versions of papers published by the $B M J$, the New Journal of Physics, and the British Journal of Educational Technology (after 2015). Details on how to create and submit such abstracts can be found at http://authorservices.taylorandfrancis.com.

\section{READABLE ABSTRACTS}

Readable abstracts normally precede or follow traditional abstracts in a paper and give a lay person's account of the content to follow. Examples can be found in the British Journal of Learning Disabilities. Here, the headings of a structured abstract are typically replaced with enumerated or bulleted text, using sentences like: " 1 . We wished to find out whether..." and "2. We worked with..."

I find it difficult to imagine why there needs to be both a readable abstract and a traditional or structured one when a readable one would do, but perhaps this is a useful way of presenting both readable and complex text for different kinds of readers.

Readable abstracts appear to be part of a more general approach to providing more information about what a reader might gain from reading a paper. Authors in the $B M J$, for instance, provide two paragraphs in addition to the main abstract with the heading, "What is already known on this topic" and "What this study adds." Curiously, this information appears at the end of the articles. Similar information usually appears up front on the second page of articles in the British Journal of Educational Technology, with the heading "Practitioner Notes." These notes are presented in a bulleted form under three headings: "What is already known about this topic"; "What this paper adds"; and "Implications for practice and/or policy."

\section{TWEETABLE ABSTRACTS}

Some journals (e.g., Methods in Ecology and Evolution and BJOG: An International Journal of Obstetrics and Gynaecology) now require authors to produce a tweetable abstract as well as a traditional one. The aim, presumably, is to facilitate the rapid dissemination of the contents of the article to a wider audience. Tweetable abstracts contain the essence of a study in less than 110-120 characters. A fabricated example reads, for example, "A review of 400 studies shows a significant superiority for structured over traditional abstracts." Undoubtedly, tweetable abstracts will ensure that the key contents of an article will be disseminated much more rapidly and more widely than is the case traditionally. 


\section{REFERENCES}

1. Hartley J. Current findings from research on structured abstracts. J Med Libr Assoc. 2004 Jul;92(3):368-71.

2. Hartley J. Current findings from research on structured abstracts: an update [comment and opinion]. J Med Libr Assoc. 2014 Jul;102(3):146-8. DOI: http://dx.doi.org/10. 3163/1536-5050.102.3.002.

\section{AUTHOR'S AFFILIATION}

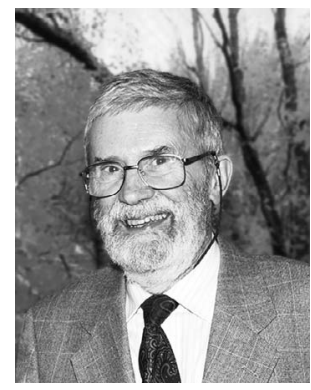

James Hartley, PhD, j.hartley@ keele.ac.uk, Emeritus Professor, School of Psychology, Keele University, Staffordshire, ST5 5BG, United Kingdom

\section{Health sciences librarians off the radar}

\author{
Sue Espe, BBA, MLIS, AHIP \\ See end of article for author's affiliation.
}

The library environment has undergone tremendous change through the years and with that change, the profession has had to adapt. These seemingly simple phrases written more than forty years ago continue to be as meaningful and applicable today as they were when written: "like everything else, libraries and library methods are changing at an increasing rate," and "'new things' have been and are happening in your field, and, indeed, some of these new trends seem to have come upon us quickly and, in some instances, have become drastic enough to threaten the very existence of the relatively small, professionally-supported medical library" [1]. Many health sciences libraries have adapted to accelerated technological advancements, fragmented collection acquisition models, altered space allocations, and modified user viewpoints. Particularly, health sciences libraries in hospitals have been profoundly impacted with library closures, bringing forth new thoughts related to value, adaptation, and change. In essence, this seemingly new trend of closures experienced today may not be so new after all, and the question that must be asked is, how has the health sciences hospital library continued to survive through the years?

One current unspoken, perhaps unconscious, survival tactic for many health sciences libraries involves working off the radar to avoid detection by administrators, who have the power to enforce decisions such as to forever close a library. In a business organization, there may be one ideal time to be off the radar and that occurs during the phase of bringing a new product to market. At that time, it is preferable to be off the radar of competitors, so they have little or no notice to attempt to copy the launched product, but once past the product introductory stage, it is best to be assertive by actively marketing the product. A prevailing business tactic for libraries to utilize is adherence to a marketing strategy that highlights remarkable operations in order to build strong business administrator and user relationships.

Having a strategic marketing plan is a practical tactic, helping to create a low-anxiety work environment that helps to assuage staff's debilitating fear of library closure or an underlying desire for the library to become invisible. Strategic marketing plans are a powerful tactic to lay the directional path to begin thinking with the mindset of a business organization. Marketing through a realistic analysis or strategic plan not only provides crucial promotion of exemplary library products and services, but also comforts staff who will know that steps are being considered to initiate long-term library survival. It is stated in psychological studies that "avoiding information is closely linked to feelings of anxiety and fear, as well as to other cognitive and emotional variables like perceptions of treatment efficacy, selfefficacy, and locus of control (the degree to which one's fate is governed externally versus controlled by one's self)" [2]. Being off the radar may provide comfort as a coping method against presumed 